\title{
Modelling and Motion Analysis of a Pill-Sized Hybrid Capsule Robot
}

\author{
M. Nazmul Huda ${ }^{1}$ (D) Pengcheng Liu ${ }^{2} \cdot$ Chitta Saha ${ }^{3} \cdot$ Hongnian $\mathrm{Yu}^{4}$
}

Received: 2 August 2018 / Accepted: 31 January 2020 / Published online: 19 March 2020

(C) The Author(s) 2020

\begin{abstract}
This paper presents a miniature hybrid capsule robot for minimally invasive in-vivo interventions such as capsule endoscopy within the GI (gastrointestinal) tract. It proposes new modes of operation for the hybrid robot namely hybrid mode and anchoring mode. The hybrid mode assists the robot to open an occlusion or to widen a narrowing. The anchoring mode enables the robot to stay in a specific place overcoming external disturbances (e.g. peristalsis) for a better and prolonged observation. The modelling of the legged, hybrid and anchoring modes are presented and analysed. Simulation results show robot propulsions in various modes. The hybrid capsule robot consisting four operating modes is more effective for the locomotion and observation within GI tract when compared to the locomotion consisting a single mean of locomotion as the hybrid robot can switch among the operating modes to suit the situation/task.
\end{abstract}

Keywords Hybrid capsule robot · Capsule endoscopy · In-vivo diagnosis · Legged mode · Legless mode · Anchoring mode $\cdot$ Modelling $\cdot$ Medical robot

\section{Introduction}

Miniature robots/robotic assistants show promise for a better and minimally invasive diagnosis and interventions.

M. Nazmul Huda

nazmul.huda@coventry.ac.uk;

nazmul.huda@brunel.ac.uk

Pengcheng Liu

pengcheng.liu@york.ac.uk

Chitta Saha

ab3135@ coventry.ac.uk

Hongnian Yu

H.Yu@napier.ac.uk

1 Electronic and Electrical Engineering, Department of Electronic and Computer Engineering, College of Engineering, Design and Physical Sciences, Brunel University London, Uxbridge, UB8 3PH, UK

2 Department of Computer Science, University of York, Deramore Lane, York, YO10 5GH, UK

3 School of Computing, Electronics and Maths, Coventry University, Coventry, CV1 5FB, UK

4 School of Engineering and the Built Environment, Edinburgh Napier University, 10 Colinton Road, Edinburgh, EH10 5DT, UK
Researches in miniature mobile robots for abdominal cavity interventions and self-propelling capsule endoscope (CE) to diagnose GI (gastrointestinal) tract diseases are rising [1-7]. Currently, available CE is passive and moves by utilising natural GI peristalsis and gravity. It cannot control its movement and orientation, cannot reverse the direction and cannot stop at a certain place for a long and detailed observation. Furthermore, it cannot distend the tissue when it is necessary especially in the colon to have a better view of the interior surface [8]. Thus, the diagnostic outcome of the $\mathrm{CE}$ is not fully reliable and it plays a complementary role with traditional probe endoscopy (PE) rather than replacing it. Researches are ongoing to add mobility to CE to increase the reliability, performance and to add functionalities such as biopsy, drug delivery, surgery etc. Mobile robots designed for the abdominal cavity, as well as GI tract, can be divided based on the propulsion mechanism used as 1) external propulsion robot (i. magnetic propulsion $[2,4,9]) 2$ ) internal propulsion robot (i. wheeled $[10,11]$ ii. legged $[1,8]$ iii. internal reaction force propulsion robot $[12,13]$ iv) vibratory actuation robot $[14,15])$ and 3 ) hybrid propulsion robot [16].

Magnetic propulsion is performed using magnet/ferromagnetic material inside the capsule which is controlled by external magnets or solenoid. The effective control is not possible for this type of propulsion and also it cannot distend the tissue for better view [16]. Moreover the use of large 
external magnet makes the system expensive. The sharp edges of legs or wheels of the legged/wheeled robots create the risk to injure the tender GI tract and internal organs. Moreover, a substantial amount of energy is required which is either supplied by batteries or tethered external sources $[8,10,11]$. The internal reaction force propulsion robot utilises the reaction force of an inner mass moving back and forth. It has no external legs or wheels which is a very good feature for in-vivo robots. However, it cannot distend the tissue for better observation [12]. A hybrid locomotion was developed in [16] where internal legged actuation mechanism and external magnetic dragging is combined. Here the legged mechanism is limited. It can lift the tissue in a collapsed region and can move the robot slightly forward. This propulsion mechanism still has the disadvantage of being expensive because of the large external magnet.

This paper is a further development of a hybrid capsule robot based on the idea in [17] which combines both internal reaction propulsion mechanism (capsule/legless propulsion) and legged propulsion mechanism. This paper proposes two new modes of operation namely hybrid mode and anchoring mode. The robot with four modes of operation is more suited for locomotion within a GI tract of the human body when compared to robots having only a single mean for propulsion. The most appropriate mode of operation of the hybrid robot can be selected to minimise/remove the chance of causing harm to the vessels through which the robot passes.

The contributions of this paper are: a) extending the operating modes of hybrid robot of [17] and adding two modes namely hybrid mode and anchoring mode where the existing actuators are utilised for executing the proposed modes b) developing the methods of moving the robot within a tubular environment in legged mode, hybrid mode and anchoring mode c) modelling of the robot in legged mode, hybrid mode and anchoring mode and d) simulation of the robot in legged mode and hybrid mode.

This paper is structured as follows. Section 2 presents the hybrid robot design. The working principles of the robot in various modes are described in Section 3. Section 4 presents the robot modeling in various modes whereas Section 5 presents the robot simulation in various modes. Section 6 presents the rationale of using four operating modes. Finally Section 7 presents the conclusion and future works.

\section{Hybrid Robot Design}

This paper presents further development based on the idea in [17]. Figure 1a and $\mathrm{b}$ show the hybrid capsule robot design and a partially exploded perspective view of the hybrid capsule robot respectively. The hybrid robot has two sets of projecting legs which can be engaged/disengaged with the cylindrical rod of the linear motors by two grippers.

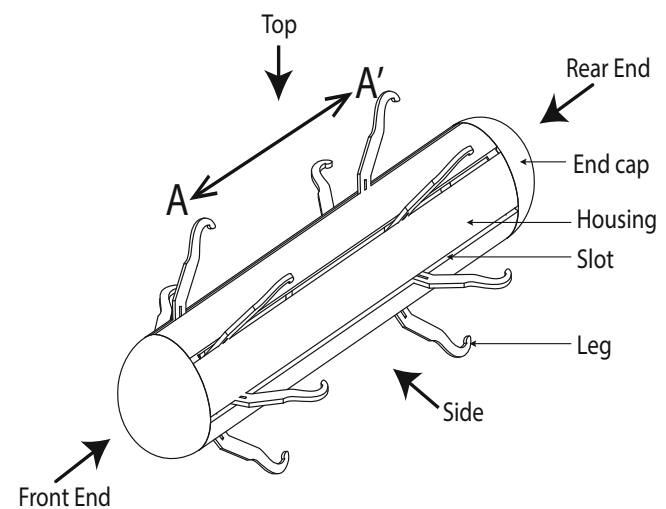

(a)

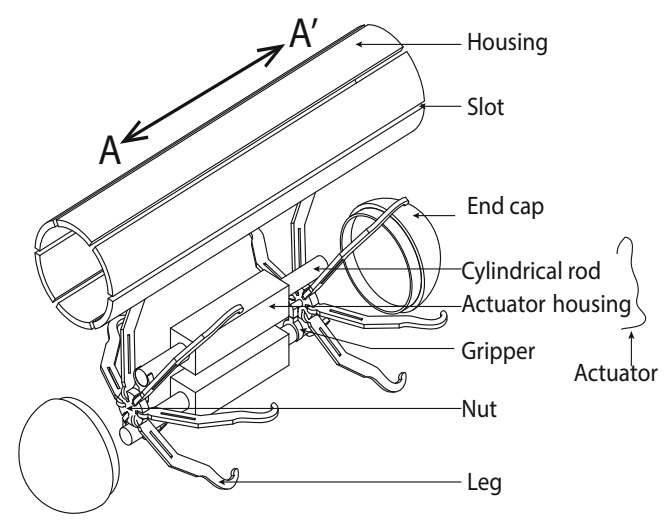

(b)

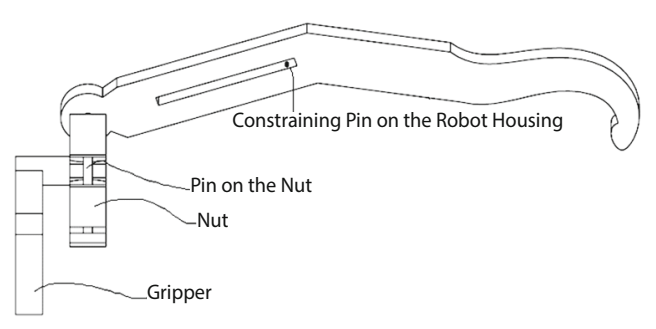

(c)

Fig. 1 Hybrid robot design (modified from [17]) a A perspective view of the hybrid robot $\mathbf{b}$ A partially exploded perspective view of the hybrid robot of Fig. 1a where the leg-sets are coupled with the cylinder rods c Leg-nut-gripper assembly showing one leg

The legs can be operated (open and close) by controlling the cylindrical rod movement. Figure 1c presents leg-nutgripper assembly showing one leg.

\section{Working Principle}

The hybrid capsule robot has four modes of operation: legless motion mode, legged motion mode, hybrid motion mode and anchoring mode. Same actuators are used to perform the operations in all the modes. 


\subsection{Legless Mode}

This is the primary propulsion mode. In this mode, the cylindrical rods act as inertial masses (IMs) to generate propulsion. The leg-sets are disengaged from the cylindrical rods and retracted inside the robot body. Thus the movement of the cylindrical rod does not cause any movement of the leg-sets. By controlling the acceleration of the cylindrical rods, the robot can i) move forward or backward and ii) rotate clockwise or counterclockwise. In the legless mode, the hybrid robot motion can be compared with the motion of the 2D capsubot described in the paper [18] of the first author. The working principle in legless mode is the same as described in [18]. The mass of the leg-nut-gripper assembly is added to the mass of the robot. The features of legless mode are: i) primary motion mode, ii) moved by the internal reaction force, iii) legs are folded inside the robot body, iv) no external moving parts, v) hermetically sealable, vi) any suitable outer structure is possible, and vii) suitable for applications inside the human body. In the legless mode, the robot is an underactuated system and nonlinear $[19,20]$ and, a behaviour based control has been presented in [18] to control this nonlinear underactuated system.

\subsection{Legged Mode}

This is the secondary propulsion mode. This mode is only activated when the robot can not pass a path using legless mode. In the legged mode (Fig. 2a), the leg-sets are connected with cylindrical rods through the grippernut assemblies. When the cylindrical rod moves linearly, the corresponding gripper-nut assembly moves linearly with it. However, the legs rotate and slide with respect to the constraining pins which are fixed on the robot cover. The leg-movements (opening and closing) are repeated to enable the robot to move. The legs can be operated (open and close) using the following control sequences so that the robot only moves in the forward direction.

- Cycle 1: At the beginning of the legged locomotion, both the leg-sets remain closed.

- Step 1: The rear leg-set opens from closed position. During this step, the robot experiences a small backward force from the reaction of the surrounding environment and it moves backward though very small.

- Step 2: The front leg-set opens from closed position. The robot experiences a small backward force. But as the hook of the rear leg-set locks the robot and opposes any backward movement, the robot remains stand-still.

- Step 3: The front leg-set closes from opened position. The robot experiences a forward force from the reaction of the surrounding

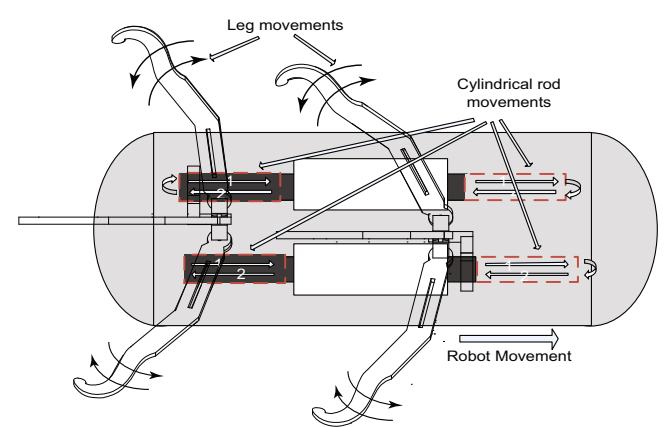

(a)

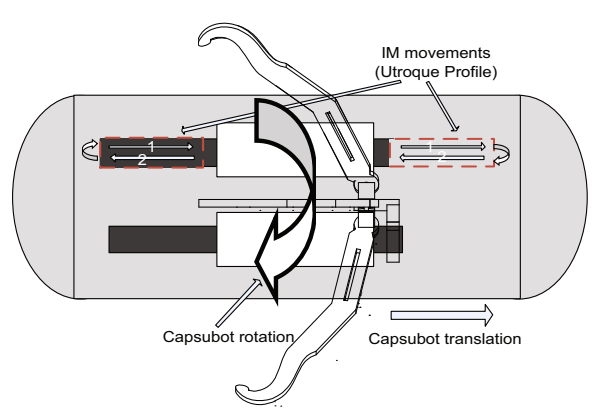

(b)

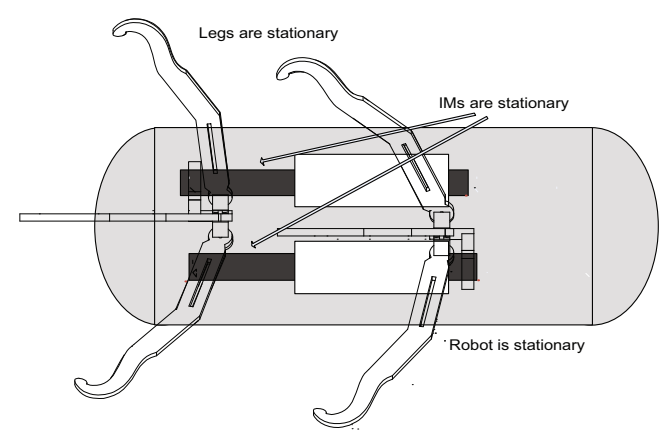

(c)

Fig. 2 Legs and cylindrical rods in various modes a Legs and cylindrical rods' movements in legged mode b Legs and cylindrical rods' movements in hybrid mode (translation-clockwise) c Stationary legs and cylindrical rods in anchoring mode

environment and it moves forward. Because of the hook-like structure, the opened rear leg-set creates very low resistance in the forward movement of the robot.

- Repeated cycle: By repeating steps 2 and 3 the robot moves forward.

The features of legged mode are: i) this is a secondary motion mode, ii) the legs come out from the robot body, and iii) the robot can pass occlusions or narrowing using this mode.

\subsection{Hybrid Mode}

In this mode, one of the leg-sets is kept always open and other leg-set is disengaged from the cylindrical rod 
and retracted inside the robot body. The free cylindrical rod is operated in legless mode. In hybrid motion mode, one of the actuators keeps one leg-set open to make a path for the robot and the other actuator works in legless motion mode to provide force to move the robot forward. It provides 'hammer blow - a very hard hit' to the robot and assist it to open an occlusion or to widen a narrowing. Thus, the features of the hybrid mode are: i) secondary motion mode ii) one linear actuator is arranged to keep one leg-set open iii) the other linear actuator of the robot operates in legless propulsion mode iv) legless propulsion is employed to hammer the consequently wedgeshaped robot (because of the opened leg-set) into the occlusion.

The hybrid motion can be divided into two types: 1) Hybrid translation-anti-clockwise rotation, and 2) Hybrid translation-clockwise rotation.

\subsubsection{Hybrid translation-anti-clockwise rotation}

The first leg-set is kept open and second cylindrical rod (inertial mass/inner mass - I $M_{2}$ ) follows the acceleration profile presented in [18]. The reaction force urges the robot to move forward. Moreover, as the reaction force does not go through the mass centre of the robot, it creates a torque with respect to the mass centre of the robot. The torque urges the robot to rotate counter-clockwise.

\subsubsection{Hybrid translation-clockwise rotation}

The second leg-set is kept open (Fig. 2b) and first cylindrical rod (inertial mass/inner mass - $I M_{1}$ ) follows the acceleration profile presented in [18]. The reaction force urges the robot to move forward. Moreover, as the reaction force does not go through the mass centre of the robot, it creates a torque which urges the robot to rotate clockwise.

\subsection{Anchoring mode}

In anchoring mode (Fig. 2c), the robot stays in a fixed position to do a certain task (e.g. delivering treatments, taking video for a long time for better observation). The actuators are used to keep both the leg-sets open. The actuators oppose any movement tendency of the leg-sets by any external force such as visceral peristalsis. The features of the anchoring mode are i) the robot does not move and ii) both the leg-sets are kept open to anchor the robot in a fixed position to perform a task (e.g. take video, deliver treatment).

\section{Modelling of the Hybrid Robot}

\subsection{Modelling of the Legless Mode}

The legless mode of the hybrid robot can be compared with the 2D capsubot presented in the paper [18] of the first author. The reader is referred to [18] for the modelling of the legless mode.

\subsection{Modelling of the Legged Mode}

By controlling the movements of the cylindrical rods the leg-sets can be opened and closed. The leg has a good contact with the surrounding environment (colon wall of GI tract) while the leg-opening is between $140^{\circ}-110^{\circ}$ [21]. Thus, the working angle is kept between $140^{\circ}-110^{\circ}$ in this paper. The closing is defined as moving the leg-set from legopening $140^{\circ}$ to $110^{\circ}$ as shown in Fig. 3a and b. The opening is defined as moving the leg-set from leg-opening $110^{\circ}$ to $140^{\circ}$ as shown in Fig. 3a. In one cycle the leg performs closing and opening i.e. moves from $140^{\circ}$ to $110^{\circ}$ and then returns to $140^{\circ}$ from $110^{\circ}$. To help the reader to follow the modelling of the legged mode a notation list is provided in Table 1.

\subsubsection{When the leg-set is closing from $140^{\circ}$ to $110^{\circ}$ and leg-tips have no contact}

Figure 3a shows the scenario where the leg-set is closing from $140^{\circ}$ to $110^{\circ}$ and leg-tips have no contact with the surrounding. Here the cylindrical rod moves towards left from A' to A" position, $\theta$ changes from $140^{\circ}$ to $110^{\circ}$, the leg moves from red dotted to blue solid position and the legtip moves from C' to C" position. The position of leg-tip for Fig. 3a:

$$
\begin{aligned}
& x_{\text {leg-tip }}=l_{1} \cos (\theta)+l_{2} \cos (\theta+\delta)+x_{m}, \\
& y_{\text {leg-tip }}=l_{1} \sin (\theta)+l_{2} \sin (\theta+\delta)+y_{m},
\end{aligned}
$$

where $\delta=$ constant $=-15^{\circ}, l_{1}=4 \mathrm{~mm}$ and $l_{2}=8 \mathrm{~mm}$.

$$
\begin{aligned}
\theta\left(x_{m}\right) & =\tan ^{-1} \frac{l_{1} \sin \left(\theta_{M}\right)}{l_{1} \cos \left(\theta_{M}\right)-x_{m}}, \\
x_{M} & =0 .
\end{aligned}
$$

- From Eqs. 1 and 3 if $\theta=\theta_{M}$ then $x_{m}=0$ and $x_{\text {leg-tip }}=-7.6528 \mathrm{~mm}$.

- Similarly from Eqs. 1 and 3 if $\theta=\theta_{m}$ then $x_{m}=$ $-2.1284 \mathrm{~mm}$ and $x_{\text {leg-tip }}=-4.1937 \mathrm{~mm}$. 


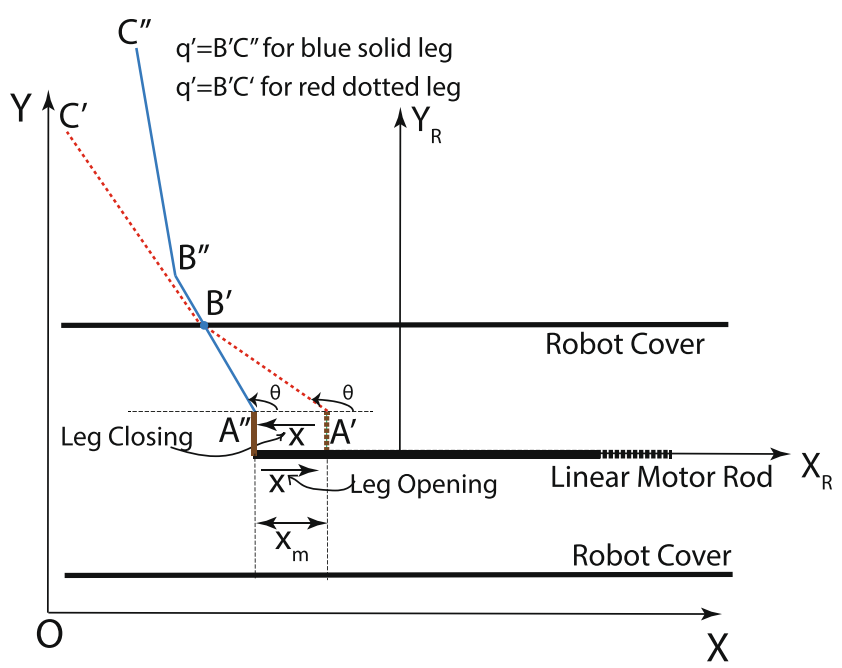

(a)

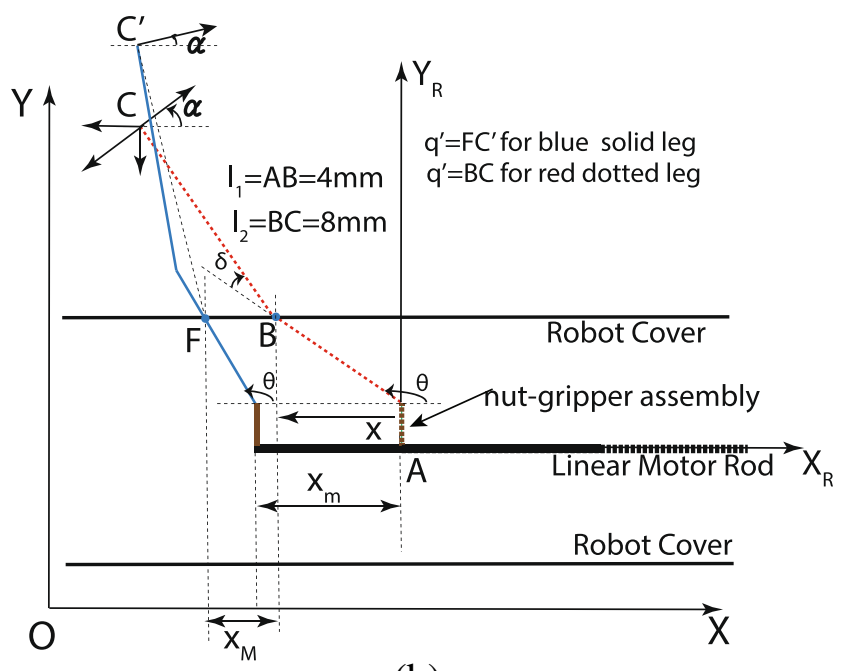

(b)

Fig. 3 a Leg closing and opening when the leg is not facing any obstacle - the robot does not move b Leg closing: the leg (red dotted and blue solid) in two positions $\left(140^{\circ}\right.$ and $\left.110^{\circ}\right)$ when the robot moves

\subsubsection{When the Leg-Set Closes and the Leg-Tips have Contact with the Surrounding}

This section presents the modelling of the legged mode when the leg-set closes from 140 degrees to 110 degrees and the leg-tips have contact with the cylindrical surrounding such as colon wall. Figure 4a shows the force balance. The linear motor housing applies $F_{a c t}$ force on the cylindrical rod and tries to move it towards left. $f_{m}$ is the friction which opposes this movement tendency. Through lever action (pin on the slot of the robot cover of each leg works as a fulcrum and forms a lever) each leg-tip applies $F_{l e g}$ force on the colon wall. The reaction by the colon wall on the leg-tip is $R_{\text {colon }}=-F_{\text {leg }}$. The rod applies " $-F_{\text {act }}$ " reaction force on the linear motor housing which is attached to the outer
Table 1 Description of the notations used

\begin{tabular}{|c|c|}
\hline Notation & Description \\
\hline$F_{\text {act }}$ & Force on the cylindrical rod by the Motor housing \\
\hline$F_{l e g}$ & Force on the colon wall of the GI tract by the leg-tip \\
\hline$n$ & Number of legs in each leg-set \\
\hline$l_{1}$ & Length of the first link of the leg \\
\hline$l_{2}$ & Length of the second link of the leg \\
\hline$\theta$ & Angle between the first link and the robot body \\
\hline$\theta_{M}$ & Maximum leg-opening, $140^{\circ}$ \\
\hline$\theta_{m}$ & Minimum leg-opening, $110^{\circ}$ \\
\hline$\delta$ & Angle between the first and second links (constant) \\
\hline$p^{\prime}$ & $\begin{array}{l}\text { Straight line distance between the constraining } \\
\text { pins (on the cover and on the nut) }\end{array}$ \\
\hline$q^{\prime}$ & $\begin{array}{l}\text { Straight-line distance between the constraining pin on } \\
\text { the cover and leg-tip (contact point with the surrounding) }\end{array}$ \\
\hline$x_{l e g-t i p}$ & Horizontal position of the leg-tip \\
\hline$y_{l e g-t i p}$ & Vertical position of the leg-tip \\
\hline$\left(x_{F}, y_{F}\right)$ & Position of the pin on the robot cover, $\mathrm{F}$ \\
\hline$\left(x_{m}, y_{m}\right)$ & Position of the cylindrical rod (inertial mass) \\
\hline$\left(x_{M}, y_{M}\right)$ & Position of the robot \\
\hline
\end{tabular}

cover of the robot. The rod and the robot are still stationary. The force on the colon wall by the leg-tip is given by:

$F_{\text {leg }}=-\frac{1}{n}\left(F_{a c t}-f_{m}\right) \sin (\theta) \frac{p^{\prime}}{q^{\prime}}$,

where,

$f_{m}=\sin \left(\dot{x}_{m}\right) \mu_{m} m g$

$p^{\prime}=\frac{p}{\sin (\theta)}$,

$q^{\prime}=\sqrt{\left(y_{\text {leg-tip }}-y_{F}\right)^{2}+\left(x_{\text {leg-tip }}-x_{F}\right)^{2}}$.

When all the components of the robot and the robot are stationary, there is a force balance. The vertical component of the reaction force of the surrounding environment such as colon wall on each leg is given by the following equation.

$F_{V}=R_{\text {colon }} \sin (\alpha)$.

There are three pairs of legs in each leg-set. As each pair cancels each others vertical component of reaction forces, the vertical components do not have any impact on the robot movement. Horizontal forces contributes to the robot movement. Following two forces are acting on the leg horizontally (towards left in Fig. 4a). Horizontal force on the leg by the rod $\left(F_{H_{1}}\right)$ and by the surrounding such as colon wall $\left(F_{H 2}\right)$ are given by the following equations respectively.

$F_{H 1}=\frac{1}{n}\left(F_{a c t}-f_{m}\right)$,

$F_{H 2}=R_{\text {colon }} \cos (\alpha)$.

As the leg is stationary, the pin on the robot cover (fulcrum of the lever) must apply a horizontal force $\left(F_{H}\right)$ 


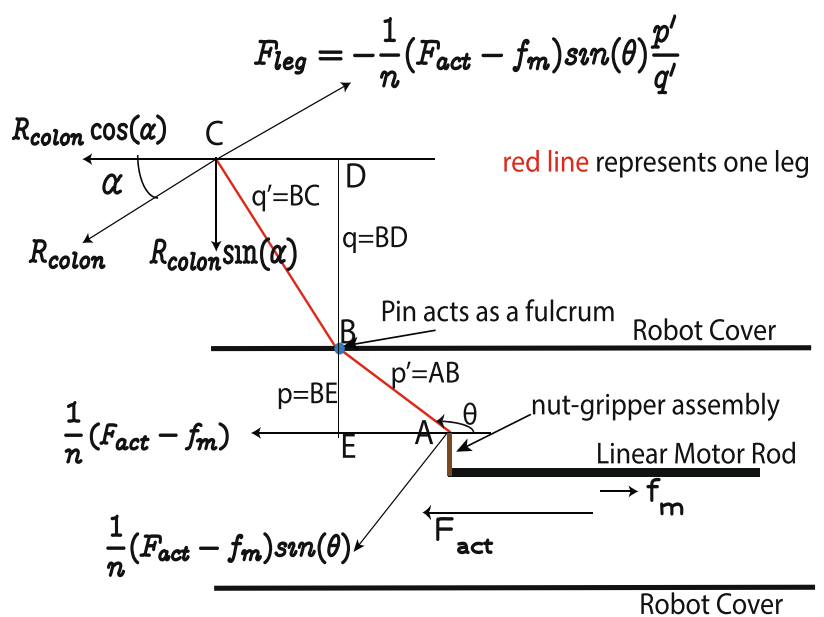

(a)

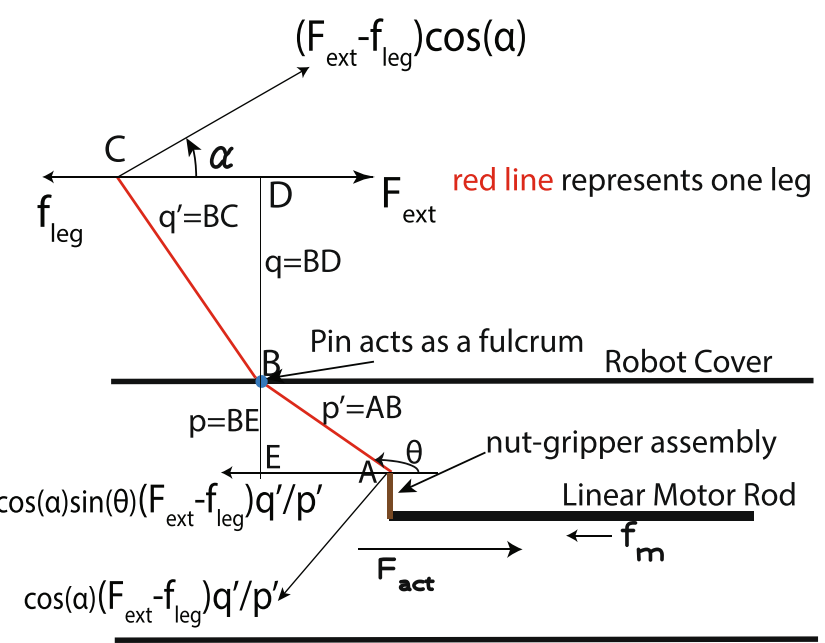

(b)

Fig. 4 Acting forces for one leg a Acting forces in the legged mode when the robot is stationary b Acting forces for one leg when $F_{\text {ext }}$ exceeds the limiting value of $f_{\text {leg }}$ (applicable to both hybrid and anchoring mode)

(towards right in Fig. 4a) force on each leg given by the following equation.

$F_{H}=\frac{1}{n}\left(F_{a c t}-f_{m}\right)+R_{\text {colon }} \cos (\alpha)$.

Each leg applies $F_{H}$ reaction force (towards left in Fig. $4 \mathrm{a}$ ) on the pin on the robot cover (fulcrum). As the pin on each slot of the leg are fixed to the robot cover, the force applied on the robot/robot cover by all the legs is $n F_{H}$. This force tries to move the robot. The cylindrical rod also applies $n F_{H 1}$ force (towards right in Fig. 4a) on the robot. The total horizontal force $\left(F_{H_{\text {robot }}}\right)$ acting on the robot (towards left in Fig. 4a) is given by the following equation.

$$
\begin{aligned}
F_{H_{\text {robot }}}= & n F_{H}-n F_{H 1}, \\
\Longrightarrow & F_{H_{\text {robot }}}=\left(F_{\text {act }}-f_{m}\right)+n R_{\text {colon }} \cos (\alpha) \\
& -\left(F_{a c t}-f_{m}\right), \\
\Longrightarrow & F_{H_{\text {robot }}}=n R_{\text {colon }} \cos (\alpha), \\
\Longrightarrow & F_{H_{\text {robot }}}=-n F_{\text {leg }}, \\
\Longrightarrow & F_{H_{\text {robot }}}=\left(F_{\text {act }}-f_{m}\right) \sin (\theta) \cos (\alpha) \frac{p^{\prime}}{q^{\prime}}
\end{aligned}
$$

The dynamic equation of the robot can be written as:

$$
\begin{aligned}
F_{H_{\text {robot }}} & =f_{M}+M \ddot{x}_{M}, \\
M \ddot{x}_{M} & =\left(F_{a c t}-f_{m}-m \ddot{x}_{m}\right) \cos (\alpha) \frac{p}{q^{\prime}}-f_{M} .
\end{aligned}
$$

where

$f_{M}=\operatorname{sgn}\left(\dot{x}_{M}\right) \mu_{M} F_{N M}, F_{N M}=M g$.

$F_{H_{\text {robot }}}$ contributes to the robot movement. The robot moves when $F_{a c t}$ is large enough so that $F_{H_{\text {robot }}}$ exceeds the friction $\left(\left|f_{M}\right|\right)$ of the robot. To maintain this force, the legtips need to have contact with the colon-wall all the time. To fulfil this constraint: both the robot and the rod moves left which causes the leg-tip to stay in the same horizontal position but leg-tip vertical position changes. In one closing cycle, the rod moves left so that the angle $\theta$ changes from $140^{\circ}$ to $110^{\circ}$ and to keep the leg-tip in the same horizontal position the distance travelled by the robot in one cycle is (from Fig. 3b):

$$
\begin{aligned}
x_{M} & =\left(l_{1} \cos (\theta)+l_{2} \cos (\theta+\delta)+x_{m}\right) \text { for } \theta_{M} \\
& -\left(l_{1} \cos (\theta)+l_{2} \cos (\theta+\delta)+x_{m}\right) \text { for } \theta_{m} .
\end{aligned}
$$

When both the rod and robot move, $\theta\left(x_{m}, x_{M}\right)$ is given by:

$\theta\left(x_{m}, x_{M}\right)=\tan ^{-1} \frac{l_{1} \sin \left(\theta_{M}\right)}{l_{1} \cos \left(\theta_{M}\right)-x_{m}+x_{M}}$,

and the leg-tip position is given by:

$x_{\text {leg-tip }}=l_{1} \cos (\theta)+l_{2} \cos (\theta+\delta)+x_{m}$,

$y_{\text {leg-tip }}=l_{1} \sin (\theta)+l_{2} \sin (\theta+\delta)+y_{m}$.

- From Eqs. 10 and 11 if $x_{\text {leg-tip }}=-7.6528 \mathrm{~mm}$ and $\theta=140^{\circ}$ then $x_{m}=0$ and $x_{M}=0$.

- From Eqs. 10 and 11 if $x_{\text {leg-tip }}=-7.6528 \mathrm{~mm}$ and $\theta=110^{\circ}$ then $x_{m}=-5.5875$ and $x_{M}=-3.4591$.

In one closing cycle, the rod moves from $x_{m}=0$ position to $x_{m}=-5.5875 \mathrm{~mm}$ position; the angle changes from $\theta=140^{\circ}$ to $\theta=110^{\circ}$; the robot moves from $x_{M}=0$ to $x_{M}=-3.4591 \mathrm{~mm}$. However, the horizontal position of the leg-tip remains unchanged i.e. $x_{l e g-t i p}=-7.6528 \mathrm{~mm}$. 


\subsubsection{When the Leg-Set Opens from $110^{\circ}$ to $140^{\circ}$}

At the end of the closing cycle, the robot is stationary, front leg-set is partially open $\left(110^{\circ}\right)$ and rear leg-set is fully open $\left(140^{\circ}\right)$. The rear leg-set maintains its open position. The rod associated with the front leg-set tries to move to open the leg from $110^{\circ}$ to $140^{\circ}$. Here the forces are same as forces during leg closing (Fig. 4a) but opposite in direction. Unlike leg closing, here the leg faces little resistance while trying to move and, thus the reaction force is also small. The force $n R_{\text {colon }} \cos (\alpha)$ is not enough to move the robot and the robot remains stationary when the leg opens from $110^{\circ}$ to $140^{\circ}$ (Fig. 3a).

\subsubsection{Repeated Cycle}

To keep the robot moving the rear leg-set is kept open and, the front leg-set opens and closes repetitively. The robot moves in the 'closing cycle' and remains stationary in the 'opening cycle'.

\subsection{Modelling of the Hybrid Mode}

In this mode, the robot performs a hybrid translationrotation because of the reaction force from the IM (cylindrical rod) that moves using the acceleration profile presented in the paper [18] of the first author. As the robot moves, the legs experience an external force. The actuator that is used to keep the leg-set open, has to apply a force to balance the external force so that the leg-set remains open. Let us consider the external force on each leg is $F_{\text {ext }}$ and limiting friction of each leg is $f_{\text {leg. }}$. Figure $4 \mathrm{~b}$ shows the acting forces for one leg in hybrid mode. From Fig. $4 b$, the required force of the actuator is given by:

$F_{a c t}=-n \cos \alpha \sin \theta\left(F_{\text {ext }}-f_{\text {leg }}\right) \frac{q^{\prime}}{p^{\prime}}+f_{m}$.

The dynamic model of the robot and the IM (cylindrical rod) which works in legless mode is as follows [18]:

$$
\begin{aligned}
F_{m_{i}}-f_{m_{i}} & =m_{i} \ddot{x}_{m i} \quad i=1,2, \\
M \ddot{x} & =\left(-F_{m_{i}}+f_{m_{i}}-f_{M}\right) \cos (\phi) \quad i=1,2, \\
M \ddot{y} & =\left(-F_{m_{i}}+f_{m_{i}}-f_{M}\right) \sin (\phi) \quad i=1,2, \\
I \ddot{\phi} & =(-1)^{i}\left[\left(-F_{m_{i}}+f_{m_{i}}\right) d_{i}-M_{f}\right] \quad i=1,2,
\end{aligned}
$$

where $\mathrm{x}, \mathrm{y}$ and $\phi$ are generalised coordinates of the robot with respect to a fixed frame; $m_{i}$ and $\mathrm{M}$ are the $I M_{i}$ (cylindrical rod) mass and the robot mass respectively; $d_{i}$ is the perpendicular distance of the direction of forces $F_{m_{i}}$ and $f_{m_{i}}$ and, the axis of rotation; $f_{M}$ is the friction force on the robot; $M_{f}$ is the frictional moment of the robot about z-axis through the mass centre of the capsule robot.

The models for both the hybrid motions (1) Hybrid translation-anti-clockwise rotation and 2) Hybrid translation-clockwise rotation) are given below:

\subsubsection{Hybrid Translation-Anti-Clockwise Rotation}

Here the robot moves forward and rotates anti-clockwise. The first cylindrical rod is used to keep open the first legset. The cylindrical rod will oppose any radial movement of the leg-sets. However, the robot as a whole can move forward. The extended leg will increase the friction. Here $I M_{2}$ (second cylindrical rod) is disengaged from the leg-set to perform the legless motion. $I M_{2}$ (second cylindrical rod) follows the utroque acceleration profile presented in [18]. Equations 14-17 become:

$$
\begin{aligned}
F_{m_{2}} & -f_{m_{2}}=m_{2} \ddot{x}_{2}, \\
M \ddot{x} & =-F_{m_{2}}+f_{m_{2}}-f_{M}, \\
I \ddot{\phi} & =\left(-F_{m_{2}}+f_{m_{2}}\right) d_{2}-M_{f} .
\end{aligned}
$$

\subsubsection{Hybrid translation-Clockwise Rotation}

Here the robot moves forward and rotates clockwise. The second cylindrical rod is used to keep open the second legset. Here $I M_{1}$ (first cylindrical rod) is dis-engaged from the leg-set to perform legless motion. $I M_{1}$ (first cylindrical rod) follows the utroque acceleration profile presented in [18]. Equations 14-17 become:

$$
\begin{aligned}
F_{m_{1}} & -f_{m_{1}}=m_{1} \ddot{x}_{1}, \\
M \ddot{x} & =-F_{m_{1}}+f_{m_{1}}-f_{M}, \\
I \ddot{\phi} & =-\left(-F_{m_{1}}+f_{m_{1}}\right) d_{1}+M_{f} .
\end{aligned}
$$

\subsection{Modelling of the Anchoring Mode}

In this mode, each of the leg-set is engaged with the corresponding cylindrical rod by the gripper and the legset is kept wide open all the time. If any external force (e.g. peristalsis) tries to move the robot, the friction of the legs will stop the robot from moving. The external force is assumed to be acting uniformly on all the legs. If $F_{\text {ext }}$ is working on each leg and $f_{\text {leg }}$ is the limiting friction of each leg then:

$F_{\text {ext }} \leq f_{\text {leg }}$.

If the external force exceeds the limiting friction force of the leg i.e. when $F_{\text {ext }}>f_{\text {leg }}$, the actuators need to provide force to stop the robot from moving. Figure $4 b$ shows the 
Table 2 Parameters for hybrid robot

\begin{tabular}{llllll}
\hline$n$ & $g$ & $\delta$ & $l_{1}$ & $l_{2}$ & $m$ \\
\hline 6 & 9.8 & $-15^{\circ}$ & $4 m m$ & $8 m m$ & $25 g m$ \\
$M$ & $\mu_{m}$ & $\mu_{M}$ & $\theta_{m}$ & $\theta_{M}$ & \\
$100 \mathrm{gm}$ & 0.2 & 0.3 & $110^{\circ}$ & $140^{\circ}$ & \\
\hline
\end{tabular}

acting forces for one leg in anchoring mode. From Fig. $4 \mathrm{~b}$, the required actuator force:

$$
F_{a c t}=-n \cos \alpha \sin \theta\left(F_{e x t}-f_{l e g}\right) \frac{q^{\prime}}{p^{\prime}}+f_{m} .
$$

\section{Simulation Results and Discussion}

The simulation is performed in the Matlab/Simulink environment where a Ode45 (Dormand-Prince) solver is used with a variable step. The equations developed in the modelling section (Section 4) are applied in the simulation. The values for various parameters used in the simulation are listed in Table 2.

\subsection{Legless Mode}

The simulation for legless motion is similar to the simulation results presented in the paper [18] of the first author.

\subsection{Legged Mode}

Figure 5 shows the simulation results for the legged motion for one closing cycle. The dynamic Eqs. 7 and 8 presented in the Section 4.2 are applied to perform simulation. Figure 5a shows the force on the IM (cylindrical rod) required to generate robot movement in legged mode while the legs are closing. It shows that the force required to generate the motion is high which ranges from $-12.5 \mathrm{~N}$ to $-21 \mathrm{~N}$. Various parameters of the robot design can be modified to improve the force requirement. One scope of improvement is the ratio $q^{\prime} / p$. From Eq. 8, it can be concluded that by reducing this ratio, the required force can be reduced.

Figure $5 \mathrm{~b}$ shows the angle of the leg with the robot body while the robot and the IM (cylindrical rod) are moving. The angle decreases from $140^{\circ}$ to $110^{\circ}$. From Fig. 5a and b it can be concluded that as the leg closes the required force increases and reaches to maximum when the leg-closing, $\theta$ is $110^{\circ}$. From the Figs. 3 and 4 , it can be seen that as the leg-closing $(\theta)$ decreases $\alpha$ and $q^{\prime} / p$ increase. From Eq. 8, it can be concluded when $\alpha$ and $q^{\prime} / p$ increase the required force will increase as well.

Figure $5 \mathrm{c}$ and $\mathrm{d}$ show the IM (cylindrical rod) and the robot translation respectively. In one closing cycle, the IM

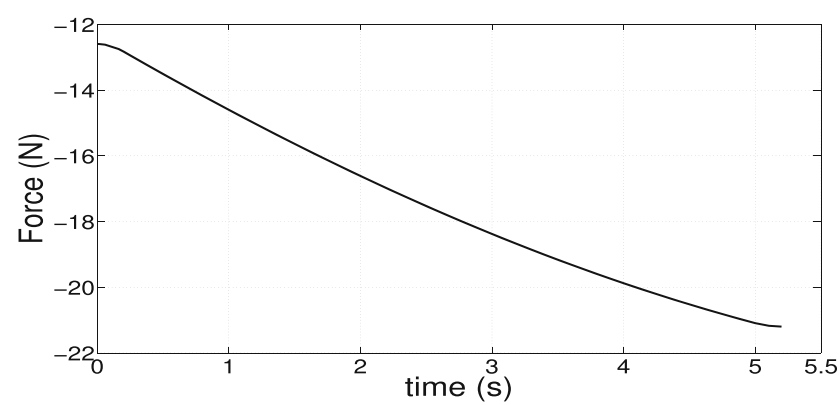

(a)

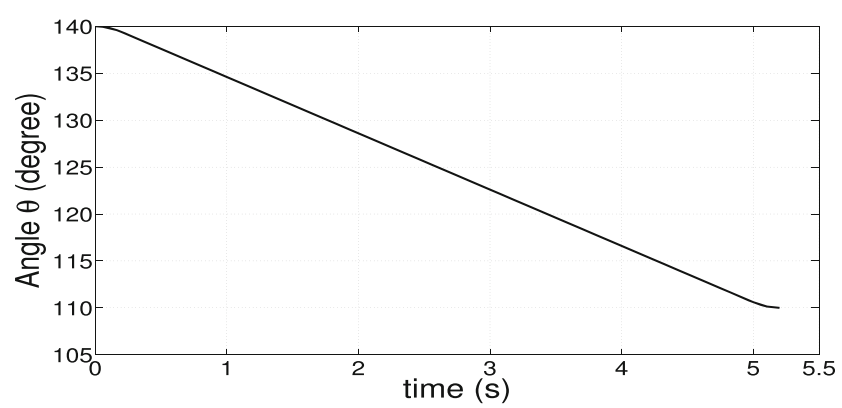

(b)

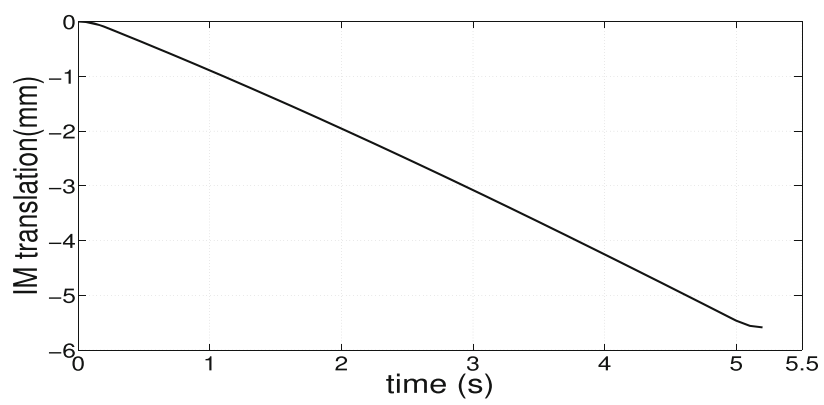

(c)

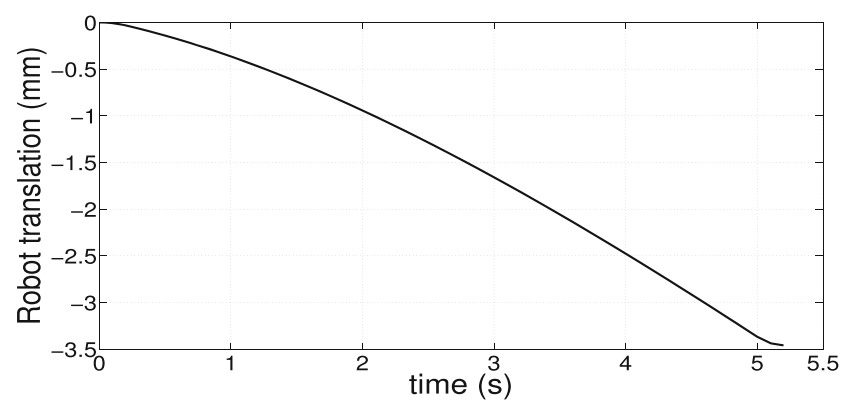

(d)

Fig. 5 Simulation results for legged movement in one closing cycle a Force applied on the IM (cylindrical rod) b Angle in the legged mode $\mathbf{c}$ IM (cylindrical rod) translation in the legged mode $\mathbf{d}$ Robot translation in the legged mode

(cylindrical rod) travels $-5.5 \mathrm{~mm}$ whereas the robot travels $-3.4 \mathrm{~mm}$. It can be seen from the Fig. $5 \mathrm{c}$ and $\mathrm{d}$ that the IM (cylindrical rod) moves faster than the robot so that there 
is always contact between the leg-tip and the surrounding environment.

\subsection{Hybrid Mode}

The dynamic equations presented in the Section 4.3 are applied to perform simulation in this section.

\subsubsection{Hybrid Translation-Clockwise Rotation}

The simulation results for hybrid translation-clockwise rotation are shown in Fig. 6. Figure $6 \mathrm{a}$ and $\mathrm{b}$ show the translation and the rotation of the hybrid robot respectively. The figures show the step-wise movement of the robot i.e. the robot moves for part of each cycle and remains stationary for the rest of the cycle. It is because of the fourstep acceleration profile [18] which the IM (cylindrical rod) follows. Figure $6 \mathrm{c}$ shows the hybrid translation-clockwise rotation in the $x-y$ plane. It is also seen that the rotation performed by the robot is small and it is less than $-2^{\circ}$ in one cycle. It can be concluded from Eq. 23 that robot clockwise rotation will increase if $d_{2}$ is increased. In Fig. 6c, the translation along the $y$-axis is negligible compared to the translation along the $\mathrm{x}$-axis.

\subsubsection{Hybrid Translation-Anti-Clockwise Rotation}

The simulation results for hybrid translation-anti-clockwise rotation are shown in Fig. 7. The figures are similar to that of Fig. 6 except that the robot rotates anti-clockwise. It can be concluded from Eq. 20 that robot clockwise rotation will increase if $d_{1}$ is increased. Similar to Fig. 6c, in Fig. 7c the translation along the $y$-axis is negligible compared to the translation along the $\mathrm{x}$-axis.

\section{Rationale of Four Modes}

The hybrid capsule robot has three motion modes (legless, legged and hybrid) and one anchoring mode. Same actuators are used for all the operating modes. The legless motion mode is the primary motion mode whereas the remaining modes are secondary. The leg-sets are disengaged from the cylindrical rods and retracted inside the robot body in the legless mode and the robot has minimal chance of causing harm to internal soft tissue. The legged mode is only activated when the robot can not pass a difficult path using legless mode. The robot returns to the legless mode once the robot passes that difficult path. The anchoring mode is activated when the robot requires to stay in a fixed position to perform a task such as delivering treatments, recording video for a long time for a detailed observation. The actuators are used to keep both the leg-sets wide open to

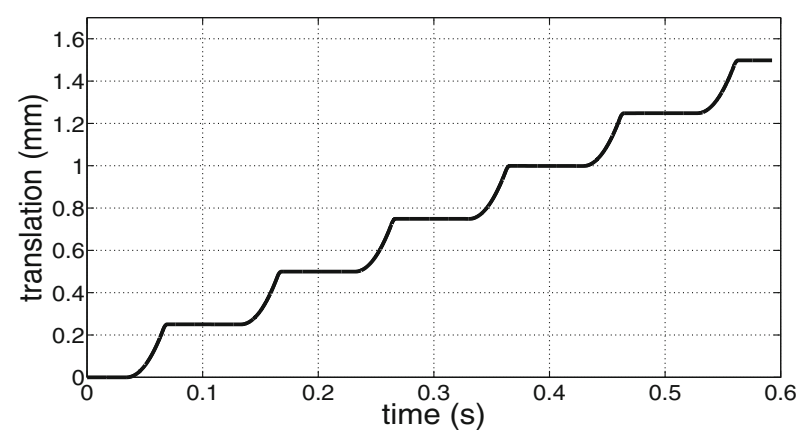

(a)

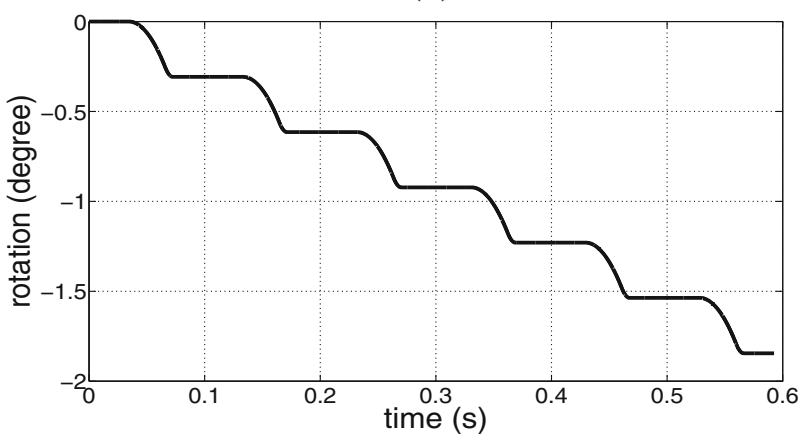

(b)

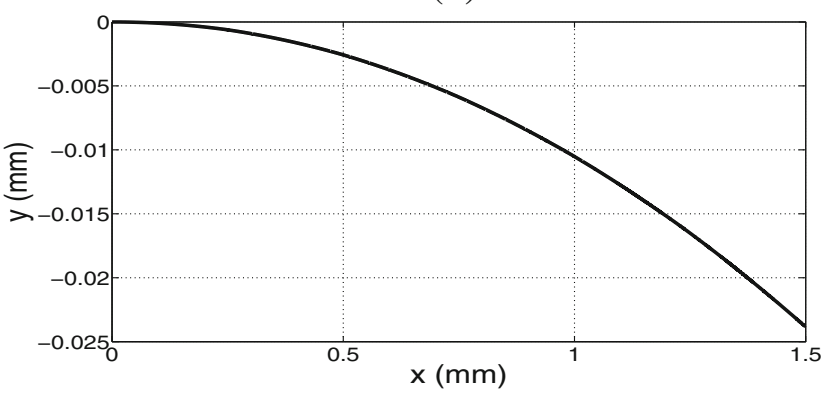

(c)

Fig. 6 Simulation results for hybrid translation-clockwise rotation a Forward translation b Clockwise rotation c Hybrid translationclockwise rotation

anchor the robot and resists the robot movement due to any external force such as peristalsis. The hybrid mode mode is activated when the robot needs to open an occlusion or to widen a narrowing. One actuator keeps one leg-set open to make a path for the robot and the other actuator works in the legless motion mode to provide force to move the robot forward to open an occlusion or to widen a narrowing. The robot can move minimally invasively in legless mode, can pass a difficult path in legged mode, can open an occlusion in hybrid mode and can stay in a fixed location overcoming external forces in anchoring mode. All these features are not available together in a robot which has only one mode. The hybrid robot is more effective for the locomotion and observation within GI tract when compared to robot with a single mode as the hybrid robot can switch among the modes to suit the situation/task. 


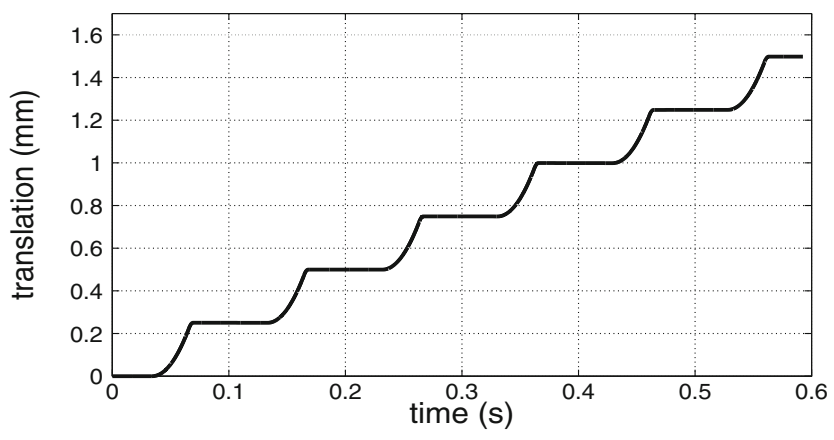

(a)

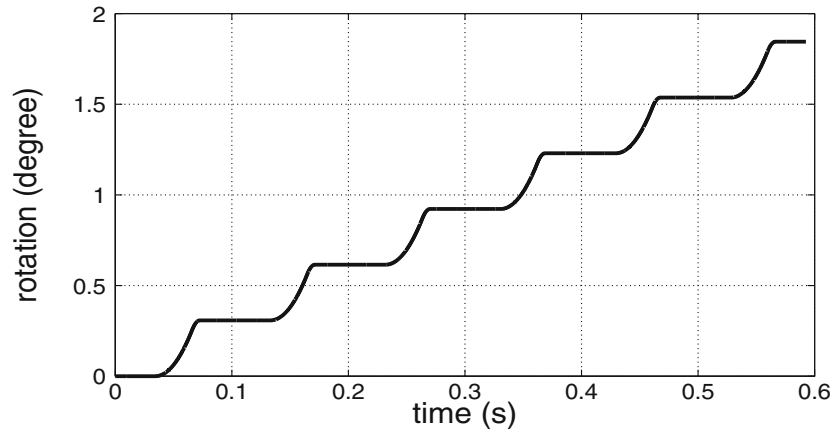

(b)

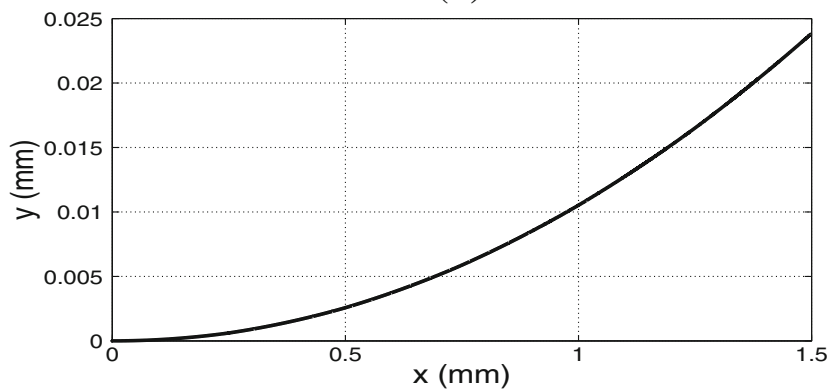

(c)

Fig. 7 Simulation results for hybrid translation-anticlockwise rotation a Forward translation b Anticlockwise rotation c Hybrid translationanti-clockwise rotation

\section{Conclusions and Future Works}

The hybrid robot is an effective solution for the in-vivo active locomotion for diagnostic purposes. Introduction of two more modes means the robot can perform four modes of operation with a set of actuators. An appropriate operating mode can be selected based on the situation/task to reduce/remove the chance of causing harm to internal tissues. The modelling of the robot for various operating modes are performed. The simulation results show the feasibility of the design and propulsion principles. In our future research we would like to optimise the robot design, in particular, we want to optimise various parameters of the leg. We also would like to develop a hybrid robot prototype and perform ex-vivo and in-vivo experimentations.
Open Access This article is licensed under a Creative Commons Attribution 4.0 International License, which permits use, sharing, adaptation, distribution and reproduction in any medium or format, as long as you give appropriate credit to the original author(s) and the source, provide a link to the Creative Commons licence, and indicate if changes were made. The images or other third party material in this article are included in the article's Creative Commons licence, unless indicated otherwise in a credit line to the material. If material is not included in the article's Creative Commons licence and your intended use is not permitted by statutory regulation or exceeds the permitted use, you will need to obtain permission directly from the copyright holder. To view a copy of this licence, visit http:// creativecommonshorg/licenses/by/4.0/.

\section{References}

1. Gao, J., Yan, G.: Locomotion analysis of an inchworm-like capsule robot in the intestinal tract. IEEE Trans. Biomed. Eng. 63(2), 300-310 (2016)

2. Natali, C.D., Beccani, M., Simaan, N., Valdastri, P.: Jacobianbased iterative method for magnetic localization in robotic capsule endoscopy. IEEE Trans. Robot. 32(2), 327-338 (2016)

3. Liu, L., Towfighian, S., Hila, A.: A review of locomotion systems for capsule endoscopy. IEEE Rev. Biomed. Eng. 8, 138-151 (2015)

4. Son, D., Yim, S., Sitti, M.: A 5-D localization method for a magnetically manipulated untethered robot using a 2-D array of hall-effect sensors. IEEE/ASME Trans. Mech. 21(2), 708-716 (2016)

5. Bao, G., Pahlavan, K., Mi, L.: Hybrid Localization of Microrobotic Endoscopic Capsule Inside Small Intestine by Data Fusion of Vision and RF Sensors. IEEE Sensors J. 15(5), 2669-2678 (2015)

6. Hawks, J., Kunowski, J., Platt, S.: In vivo demonstration of surgical task assistance using miniature robots. IEEE Trans. Biomed. Eng. 59(10), 2866-2873 (2012)

7. Sun, Z.J., Ye, B., Qiu, Y., Cheng, X.G., Zhang, H.H., Liu, S.: Preliminary study of a legged capsule robot actuated wirelessly by magnetic torque. IEEE Trans. Magn. 50(8), 1-6 (2014)

8. Valdastri, P., Webster, R.J., Quaglia, C., Quirini, M., Menciassi, A., Dario, P.: A new mechanism for mesoscale legged locomotion in compliant tubular environments. IEEE Trans. Robot. 25(5), 1047-1057 (2009)

9. Munoz, F., Alici, G., Zhou, H., Li, W., Sitti, M.: Analysis of magnetic interaction in remotely controlled magnetic devices and its application to a capsule robot for drug delivery. IEEE/ASME Trans. Mech. 23(1), 298-310 (2018)

10. Prendergast, J.M., Formosa, G.A., Rentschler, M.E.: A platform for developing robotic navigation strategies in a deformable, dynamic environment. IEEE Robot. Auto. Lett. 3(3), 2670-2677 (2018)

11. Platt, S., Hawks, J., Rentschler, M.: Vision and task assistance using modular wireless in vivo surgical robots. IEEE Trans. Biomed. Eng. 56(6), 1700-1710 (2009)

12. Yu, H., Huda M.N., Wane S.O.: A novel acceleration profile for the motion control of capsubots. In: IEEE international conference on robotics and automation (ICRA), pp. 2437-2442 (2011)

13. Huda, M.N., Yu, H., Goodwin, M.J.: Experimental study of a capsubot for two dimensional movements. In: UKACC international conference on control, pp. 108-113. Best student paper (2012)

14. Liu, P., Yu, H., Cang, S.: Optimized adaptive tracking control for an underactuated vibro-driven capsule system. Nonlinear Dyn 94, $1-15$ (2018) 
15. Carta, R., Sfakiotakis, M., Pateromichelakis, N., Thoné, J., Tsakiris, D., Puers, R.: A multi-coil inductive powering system for an endoscopic capsule with vibratory actuation. Sens. Actuator A Phys. 172(1), 253-258 (2011)

16. Simi, M., Valdastri, P., Quaglia, C., Menciassi, A., Dario, P.: Design, fabrication, and testing of a capsule with hybrid locomotion for gastrointestinal tract exploration. IEEE/ASME Trans. Mech. 15(2), 170-180 (2010)

17. Yu, H., Huda, M.N., Liu, Y., Wane, S.O.: Travelling capsule with two drive mechanisms (2013)

18. Huda, M.N., Yu, H., Cang, S.: Behaviour-based control approach for the trajectory tracking of an underactuated planar capsule robot. IET Control Theory Appl. 9(2), 163-175 (2014)

19. Sun, K., Mou, S., Qiu, J., Wang, T., Gao, H.: Adaptive fuzzy control for non-triangular structural stochastic switched nonlinear systems with full state constraints. IEEE Trans. Fuzzy Syst. 27(8), 1587-1601 (2018)

20. Qiu, J., Sun, K., Wang, T., Gao, H.: Observer-based fuzzy adaptive event-triggered control for pure-feedback nonlinear systems with prescribed performance. IEEE Trans. Fuzzy Syst. 27(11), 21522162 (2019)

21. Quirini, M., Menciassi, A., Scapellato, S., Dario, P., Rieber, F., Ho, C.N., et al.: Feasibility proof of a legged locomotion capsule for the gi tract. Gastrointestinal Endoscopy 67(7), 1153-1158 (2008)

Publisher's Note Springer Nature remains neutral with regard to jurisdictional claims in published maps and institutional affiliations.

Dr. M. Nazmul Huda received his BSc (Hons) degree in Electrical and Electronic Engineering from Bangladesh University of Engineering and Technology, Bangladesh in 2008, his MSc by Research degree in Computing Science from Staffordshire University, UK in 2011 and his $\mathrm{Ph} . \mathrm{D}$. degree in Robotics and Control from Bournemouth University, UK in 2016. At present, he is a Senior Lecturer in Electronic and Electrical Engineering at Brunel University London and supervising several $\mathrm{PhD}$ students in robotics, artificial intelligence and renewable energy. Before joining at Brunel University London, he has held several academic/research positions at Coventry University, Cranfield University, Bournemouth University, Staffordshire University and Bangladesh. He has more than ten years of experience in performing research and leading research projects in robotics, control and machine learning funded by various funding body including EPSRC and Innovate UK. He has filed a patent and published papers on flagship journals and conferences. He is a member of IET, IEEE, IEEE RAS and EPSRC associate peer review college. He has been nominated as a regular reviewer for EPSRC grants applications. He has been collaborating with internal and external academic and industrial partners and actively developing research proposals as a PI and CoI for internal and external funding calls including Horizon 2020, Wellcome Trust and High-Volume Transport. He also serves as a reviewer for many flagship journals and conferences in robotics, control and artificial intelligence including IEEE ICRA, IEEE IROS, IEEE SSRR, Autonomous Robots etc.
Dr. Pengcheng Liu received the B.Eng. degree in measurement and control and the M.Sc. degree in control theory and control engineering from Zhongyuan University of Technology, China, in 2007 and 2012, respectively, and the Ph.D. degree in robotics and control from Bournemouth University, UK, in 2017. He is currently a Lecturer (Tenured Assistant Professor) at the Department of Computer Science, University of York, UK. Before joining York, he has held several academic positions at Cardiff, Lincoln, Bournemouth and China. He is a member of IEEE, IEEE RAS, IEEE CSS and IFAC. He is also a member of the IEEE Technical Committee on Bio Robotics, Soft Robotics, Robot Learning, and Safety, Security and Rescue Robotics. He is an Associate Editor of IEEE Access and he received the Global Peer Review Awards from Web of Science in 2019, and the Outstanding Contribution Awards from Elsevier in 2017. He has published over 60 papers on flagship journals and conferences. He was nominated as a regular Funding/Grants reviewer for EPSRC, NIHR and NSFC and he has been leading and involving in several research projects and grants, including EPSRC, Newton Fund, Innovate UK, Horizon 2020, Erasmus Mundus, FP7-PEOPLE, NSFC, etc. He serves as reviewers for over 30 flagship journals and conferences in robotics, AI and control, e.g NEUNET, NODY, JINT, RNC, ICRA, IROS, RA-L, NCAA, etc. His research interests include robotics, machine learning, dynamical systems control and optimization.

Dr. Chitta Saha is currently an Assistant Professor in Electrical and Electronic Engineering at the Faculty of Engineering, Environmental and Computing, Coventry University and leads the MSc module on Alternative Energy and Smart grid where he lecturers extensively on solar PV. His current research is involved in solar PV system and its application areas, energy harvesting technologies, smart grid and electric vehicles.

During 2013-2015, he was working as a Co-PI on a 140k TSB funded energy harvesting (REGAHE10305) research project collaboration with Piezotag ((www.piezotag.com) and Severn Trent (www.stwater.co.uk). He had been a Research Associate/Fellow on a $2 \mathrm{~m}$ multi-universities EPSRC funded SCORE project (www.score.uk. com) at the University of Nottingham for four and half years.

$\mathrm{He}$ is currently acting as an Editorial board member of International Journal of Sustainable and Green Energy and supervising $4 \mathrm{PhD}$ students on solar PV and smart grid areas. In 2019, he was acting an International advisory committee member in International Conference on Recent Trends on Electronics \& Computer Science (RTECS-2019), National Institute of Technology, Silchar, India. He has published over 32 articles in leading international journals and conference with over 2300 citations and an h-index of 13 . 
Professor Hongnian $\mathrm{Yu}$ is head of research at the School of Engineering and the Built Environment, Edinburgh Napier University, has successfully supervised $20 \mathrm{PhD}$ theses and 18 Master by Research theses, and has examined over $40 \mathrm{PhD} / \mathrm{MPhil}$ students theses as both internal and external examiner. He has trained 12 post-doctoral research fellows. His research covers the two main areas: a) Robotics with applications in the rescue and recovery operations, and healthcare and 2) ICT enabled healthcare including assistive technologies in supporting elderly and people with dementia, activity recognition of elderly people. He has published over 200 journal and conference research papers. He has held several research grants worth about eight million pounds from the UK EPSRC, the Royal Society, and the European, AWM, as well as from industry. He has managed several international large consortiums as a coordinator. Examples are 1) coordinator (PI) of an EPSRC funded $158 \mathrm{~K}$ international network project on Human Adaptive Mechatronics which includes 7 Japanese partners and 7 UK partners; 2) coordinator of the EU funded 3.05 million Euro Erasmus Mundus FUSION project which has 20 international partners; 3) coordinator of the EU funded one million Euro Marie Curie project, etc. Prof Yu has strong research collaboration with partners from over 30 countries, such as China, France, Germany, Hungary, India, Italy, Japan, Romania, Thailand, and UK. He was awarded the F.C. William Premium for his paper on adaptive and robust control of robot manipulators by the IEE Council, and has received two best conference paper awards, seven best (student) conference paper awards with his research students. He has won the Gold Medal on The World Exhibition on Inventions, Research and New Technologies, INNOVA 2009, Brussels, and International Exhibition of Inventions, Geneva, Switzerland, 2010, for invention Method and device for driving mobile inertial robots; and the 43rd International Exhibition of inventions, New Techniques and Products, in Geneva, 2015. He is a member of the EPSRC Peer Review College, Fellow of the IeT, Fellow of the RSA, and senior member of IEEE. 